\title{
Fabrication and Characterization of Ultra-High Aspect Ratio Features in Gold Using the Helium Ion Microscope
}

\author{
Vincent S. Smentkowski, Radislav A. Potyrailo, Larry Scipioni*, David Ferranti* \\ General Electric Global Research Center, 1 Research Circle, Niskayuna, NY, 12309 \\ *Carl Zeiss SMT, Inc, One Corporation Way, Peabody, MA, 01960
}

Transducers with sensing features on the nanometer and smaller scales are important in chemical and biological sensing because they approach the size of the measured moieties [1]. At GE-GRC, we are developing approaches to fabricate these transducers in the laboratory using a variety of prototyping systems, including focused ion beam (FIB) [2]. Laboratory prototyping using FIB has been recognized as an attractive laboratory tool [3]. We have shown that surface contamination from the FIB nano-patterned surfaces should be removed in order to prevent changes in sensing performance [4]. Unfortunately, two significant limitations of existing FIB milling approaches are the tremendous difficulty in producing milled regions such as vias with high aspect ratio of their depth to their diameter and the deposition/implantation of $\mathrm{Ga}$ into the material being patterned. Thus a method is needed for creating the features which are needed for label-free bio-sensing and other sensing applications [5].

The Helium ion microscope (HIM) has a spot size less than $1 \mathrm{~nm}$, uses a chemically inert novel gas $(\mathrm{He})$ which does not deposit/implant any species that may contaminate the material being patterned, and is able to rapidly generate arrays of vias in Au. Hence HIM is an ideal tool to generate these ultra-high aspect ratio features. In this presentation, we will demonstrate that the Carl Zeiss ORION $^{\mathrm{TM}}$ helium ion microscope is able to generate ultra-high aspect ratio vias $(11: 1)$ with a lateral dimension of $11 \mathrm{~nm}$ by $125 \mathrm{~nm}$ deep. Additionally, the ion dose required for imaging is $1-2$ orders of magnitude less than that for milling, so the same beam can be used for inspection. We also report a novel HIM cross sectioning method that was devised and utilized to accurately measure ultra-high aspect ratio vias. The method involves eroding a $200 \mathrm{~nm}$ square hole with the bottom sloping at an angle of 45 degrees (which is about 150nm deep at the deep end) into the substrate using 1pA of current prior to milling ultra-high aspect ratio vias near the deep end of the larger, angled, crater as illustrated in FIG 1. A top down HIM image of various craters generated in Au is shown in the right panel of FIG 2.

[1] K. Shigeto, et al., Microelectronic Engineering, 83 (2006) 1471.

[2] R.A. Potyrailo, et al., Proc. Transducers '09 (2009) 2378.

[3] C. Genet, T.W. Ebbesen, Nature 445 (2007) 39.

[4] K. Dovidenko, et al., Microscopy and Microanalysis 12 (2006) 206.

[5] K.-L. Lee, et al., Biosens. Bioelectron. 24 (2008) 210. 

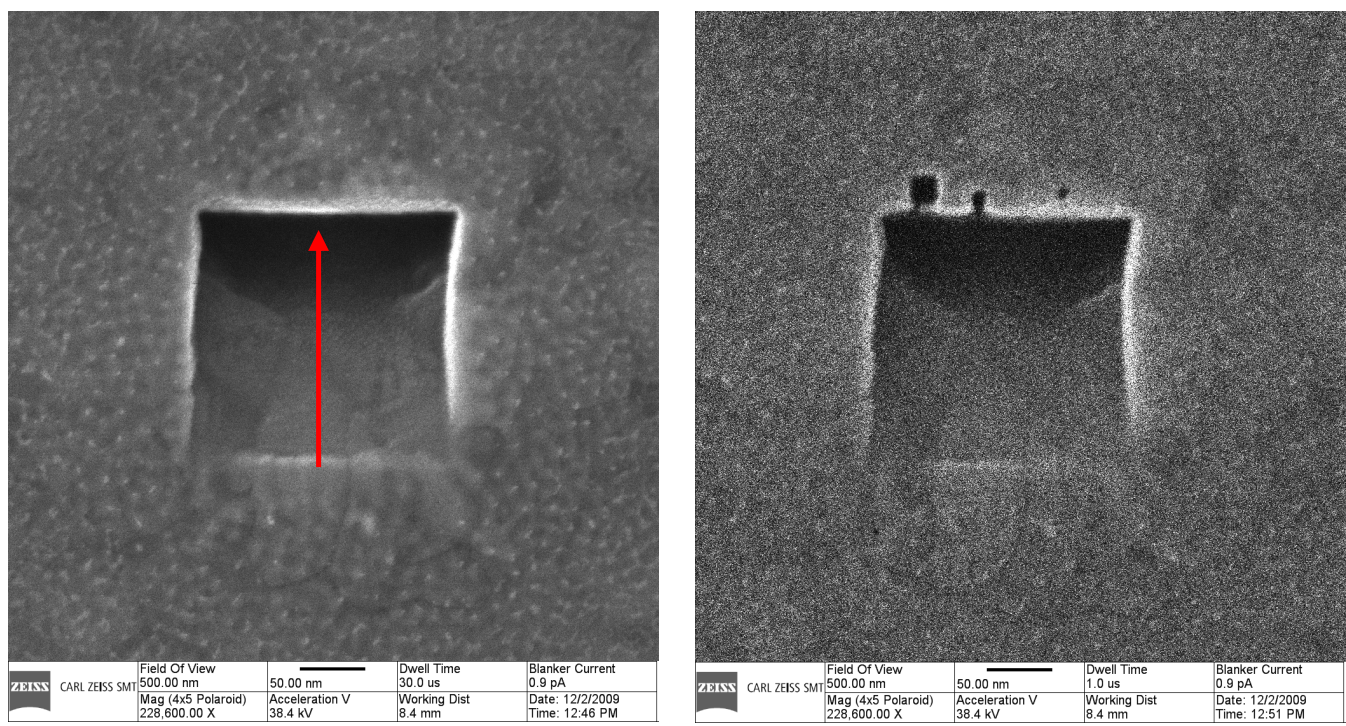

FIG. 1. The HIM image on the left shows a 200nm square crater that was milled into the gold substrate at an angle of $45 \mathrm{deg}$ (first). The HIM image on the right shows three smaller craters (25, $10,5 \mathrm{~nm})$ that were milled into Au at normal incidence after the formation of the larger crater. The erosion direction is indicated by the red arrow in the left panel.
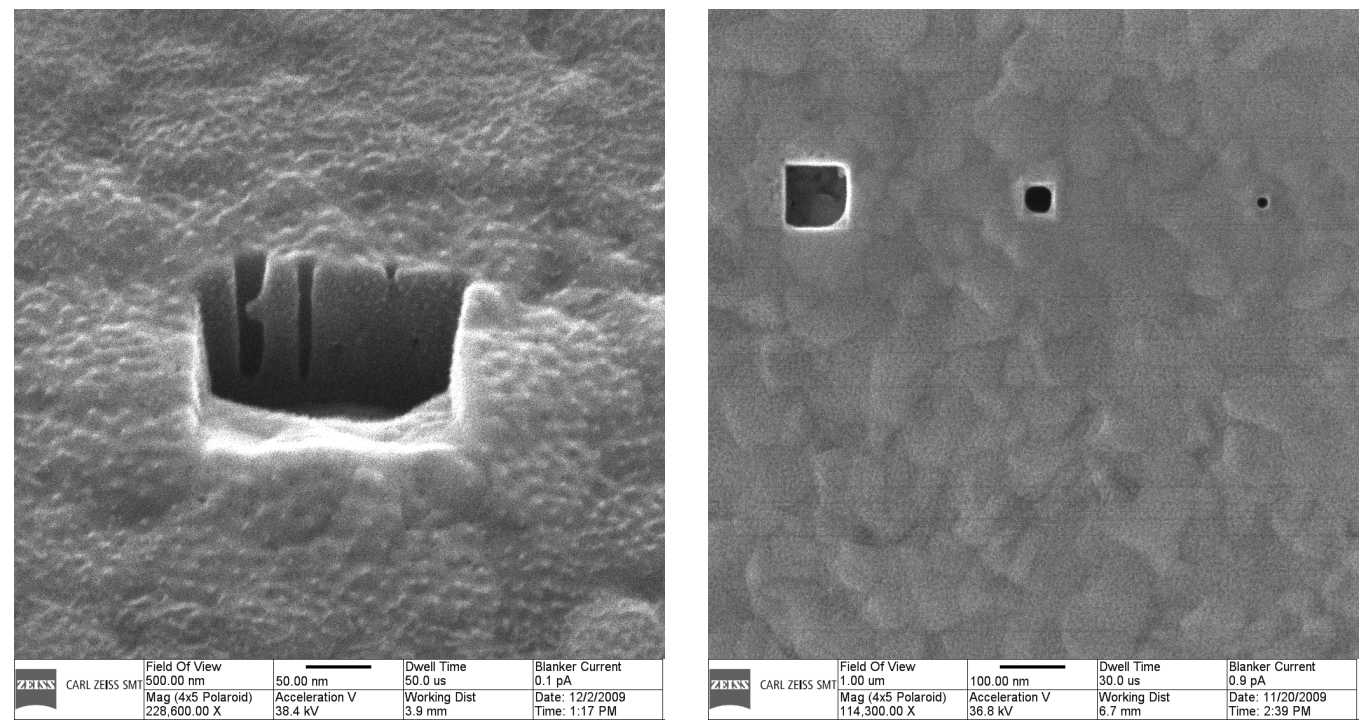

FIG. 2. The HIM image on the left was taken at a 45 degree tilt in order to visualize and measure the depth of the ultra high aspect ratio craters. The HIM image on the right was taken (at normal incidence) of three holes $(100,50$ and $25 \mathrm{~nm})$ that were milled into gold. 